\title{
FICValdivia y su posición en el cine chileno contemporáneo
}

\section{FICValdivia and its role in contemporary Chilean cinema}

\section{Sebastián González}

University of Edinburgh, Edimburgo, Escocia

sebastian.gonzalez@ed.ac.uk

https://orcid.org/0000-0001-9236-1193

\section{Resumen}

Este artículo es una revisión histórica del rol que el Festival Internacional de Cine de Valdivia ha desempeñado en el desarrollo y promoción del cine chileno contemporáneo. Analizando su programación y películas participantes desde su creación en 1994 hasta el año 2019 es posible establecer cómo el certamen se ha posicionado como el "sitio de paso" fundamental para una camada de cineastas y productores chilenos previo y durante su consolidación dentro del circuito global de festivales de cine. En más de 25 años, el FICValdivia ha experimentado cambios importantes en su línea editorial, desde una mirada ligada a la ecología, pasando por la industria y en los últimos años, bajo la dirección de Raúl Camargo, a una línea curatorial profundamente cinéfila. Desde esta posición es que el FICValdivia se ha posicionado como un punto de encuentro para la cinefilia y la comunidad del cine chileno.

Palabras clave: Festivales de Cine, Cinefilia, Programación curatorial, FICValdivia.

\begin{abstract}
This article is a historical review of the role that the Valdivia International Film Festival has played in the development and promotion of contemporary Chilean cinema. By analyzing its programming and films since its creation in 1994 until 2019, it is possible to establish how the festival has positioned itself as the crucial "site of passage" for a new generation of Chilean filmmakers and producers before and during its consolidation within the global circuit of film festivals. In over 25 years, FICValdivia has experienced significant changes in terms of its editorial line, from an ecological perspective, through an industrial frame, and, recently, under the direction of Raúl Camargo, to a deeply film-oriented curatorial line. Thus, FICValdivia has positioned itself as a meeting point for the cinephilia and the Chilean film community.
\end{abstract}

Keywords: Film Festivals, Cinephilia, Film Programming, Curatorial Practice, FICValdivia. 


\section{Introducción}

En las últimas dos décadas, Chile ha vivido una masificación de los festivales de cine como espacios de promoción para el cine chileno e internacional. En un aún incipiente circuito, existe un grupo de festivales que se destacan no sólo por su trayectoria, sino por su posicionamiento a nivel internacional, acumulando los estrenos mundiales de películas chilenas y teniendo una participación de películas relevantes para el circuito internacional. El Festival Internacional de Cine de Valdivia, popularmente conocido como FICValdivia, puede ser considerado el más importante del país y uno de los más trascendentales a nivel latinoamericano. Todos los años, la comunidad del cine chileno y relevantes críticos e invitados internacionales, se dan cita en una suerte de peregrinación cinéfila en torno al cine.

Cada año, alrededor de 10 películas chilenas se ven por primera vez en Chile durante el FICValdivia. Estas películas serán protagonistas del circuito de festivales de cine chileno y latinoamericano, teniendo una presencia masiva en las competencias y proyecciones especiales en los festivales menores. De la misma manera, muchos de sus autores serán considerados parte de la historia del cine chileno, sus películas serán exhibidas en cine clubes y estudiadas por la crítica y la academia. En 2017, el crítico de cine Robert Koehler, en un especial de la revista Sight and Sound sobre cine latinoamericano, destacaba al FICValdivia como un festival arriesgado y lo suficientemente importante para que en su ceremonia inaugural se anunciaran nuevas leyes relacionadas al cine nacional (Koehler, 2017)'. El festival ha tenido un rol fundamental en el desarrollo y la promoción del cine chileno, transformándose en un icono a nivel programático y con una clara identidad en su línea curatorial.

El presente artículo analiza cómo FICValdivia se ha convertido en un espacio fundamental de exhibición para un cine chileno contemporáneo, adquiriendo relevancia nacional e internacional gracias a su participación en la selección oficial del festival. La parte inicial de este artículo es una aproximación a las principales teorías sobre los estudios de festivales de cine, que permita realizar un análisis para comprender el desarrollo y consolidación del FICValdivia como un espacio para la cinefilia y la industria del cine chileno. La segunda parte detalla la metodología para este estudio de caso. La tercera parte dialoga con estas teorías, pero desde el punto de vista de la historia y desarrollo del festival desde su creación en 1994 hasta su versión en 2019, analizando el rol de sus distintos directores y su relación con la ciudad en que se aloja. La cuarta parte se enfoca en la relación que ha tenido el FICValdivia con el cine chileno, tanto como exhibidor, como promotor de la internacionalización del cine chileno reciente.

\section{Marco teórico}

Marijke de Valck sostiene que los festivales son "una parte esencial de la cultura cinematográfica mundial" (2016, p.9), por lo que el estudio de los festivales de cine puede entenderse como una herramienta para comprender el desarrollo de los cines nacionales, sus modelos de financiación y la percepción que tiene el público de las películas que circulan en estos espacios. En el contexto chileno, el estudio de los festivales de cine es necesario para comprender la explosión del cine chileno desde mediados de la década de 2000 desde una perspectiva que abarque la curatoría, distribución y exhibición cinematográfica. A partir de esa fecha, el cine chileno se ha consolidado en lo que Pierre Bourdieu definió como un campo de producción cultural, es decir, un área cultural que no sólo crea objetos culturales, sino que también desarrolla jerarquías e instituciones que actúan como mediadoras en los diversos ámbitos que este campo permite (Bourdieu, 1993).

A pesar de la creciente y cada vez más diversa literatura sobre festivales de cine, todavía es bastante difícil encontrar una definición de qué es un festival y la abundante literatura que se ha acumulado en la última década ha asumido como uno de sus principales objetivos la búsqueda de una definición (Vallejo, 2014a, p.20). Para el presente artículo, se usará el concepto de festival desarrollado por el sitio www.festivalesdecine.cl, que entiende estos eventos como:

Toda aquella muestra de cine y/o audiovisual que tenga una duración igual o mayor a dos jornadas (días), que se realiza de manera regular y cíclica (cada año o bianualmente), y que, además de exhi- 
bir películas de manera pública, incluya actividades que extienden la experiencia del visionado colectivo (por ejemplo, mediante charlas, paneles de discusión y otras actividades paralelas). (Peirano \& González, 2018, p. 2).

De Valck propone entender los festivales de cine "como puntos nodales de una exitosa red del cine" (2007, p.15). Para explicar esta relación, de Valck utiliza la idea desarrollada por Thomas Elsaesser de un esquema binario Europa-Hollywood "no en el sentido de oposición, sino como una relación en el contexto de los procesos de globalización" (de Valck 2007, p. 15). Elsaesser sostiene que los festivales son una red, en la que compiten entre sí por el reconocimiento, pero que al mismo tiempo son capaces de colaborar cuando encuentran un terreno común (2005). Es a través de esta relación que de Valck observa los festivales de cine como una red, donde cada uno de estos eventos actúa como un nodo, que conectan una red que forma el circuito mundial de festivales de cine en el que se incluyen todos los eventos. Estos nodos son indispensables para sostener esta red:

los festivales de cine se pueden considerar puntos de paso obligatorios porque son eventos -actores- que han llegado a ser tan importantes para la producción, distribución y consumo de muchas películas que, sin ellos, toda una red de prácticas, lugares, personas, etc. se desmoronaría (De Valck, 2007, p.36).

Es importante entender los festivales como nodos que sostienen esta red, porque los festivales han creado un circuito de distribución alternativo que permite la preservación del cine de arte y de autor, así como la promoción de películas de países más pequeños. Las películas, los actores y los agentes de la industria necesitan que los festivales sostengan esta red de distribución para su trabajo, dando a la red durabilidad y estabilidad. Sin embargo, esta red también dispone ciertas jerarquizaciones dentro del circuito. Producto de la globalización, los festivales compiten entre sí tanto internacional como nacionalmente, situándose en la lógica de las "industrias creativas", haciéndose partícipes de las cadenas de producción del cine ${ }^{2}$ y compitiendo por financiamiento y estrenos (Loist, 2016). Esta red desarrolla distintos circuitos dentro del circuito global, donde existen festivales más relevantes que otros.
En el contexto chileno, se puede apreciar que dentro del circuito nacional existen festivales que ostentan mayor relevancia internacional tanto para la comunidad del cine chileno como para la prensa y cinefilia locales. Este conjunto de festivales se componen por FICValdivia, el Festival Internacional de Documentales de Santiago (FIDOCS), Santiago Festival Internacional de Cine (SANFIC) y el Festival internacional de Cine de Viña del Mar. Este grupo de festivales ha sido denominado como eventos "internacionales de trayectoria conocida", concentrando la mayor cantidad de estrenos de películas chilenas e internacionales, organizando los principales eventos de industria en el país y generando la mayor atención de prensa nacional e internacional, siendo los cuatro eventos claves en el circuito chileno (Peirano \& González, 2018, Peirano, 2016). Sin embargo, el que ha logrado mayor relevancia a nivel internacional es FICValdivia, tanto por su línea curatorial como por su relación e identificación con la ciudad que lo alberga. Cindy Wong sostiene que las relaciones entre los festivales de cine y las ciudades, en general, sirven tanto para mejorar la consolidación de los festivales y los espacios culturales, como para posicionar a las ciudades dentro de un circuito de ciudades culturales. En sus palabras:

En la mayoría de los festivales, los funcionarios del gobierno local están presentes en los actos ceremoniales, lo que da legitimidad a los festivales y el sello de aprobación de la ciudad, a menudo con asistencia financiera. La mayoría de festivales son demasiado pequeños para beneficiar o perjudicar sustancialmente a la ciudad por su ausencia o presencia, pero junto con otros acontecimientos culturales, de otros festivales de arte de uno u otro tipo, conciertos, teatros y museos, los festivales de cine contribuyen al mosaico cultural que quieren representar las ciudades del mundo (Wong, 2011, p. 57).

En términos curatoriales y de programación, los festivales de cine cumplen un rol legitimador para directores, productores y películas. Los festivales de cine, al crear competencias, permiten que las películas ganen premios y el premio es "la forma más tangible de capital simbólico" (De Valck, 2016, p.106). Siguiendo la misma línea, James English argumenta cómo los premios dan valor y calidad al trabajo artístico. English propone que los premios y competencias generan que los nuevos y los establecidos compitan entre sí para lograr un 
mayor prestigio, al mismo tiempo que tienen que "defender o mejorar sus posiciones en el campo de la producción cultural" (English, 2008, pp.53-54). Ambos argumentos se basan en el concepto de capital cultural y simbólico, que forman parte de los diferentes tipos de capital definidos por Bourdieu. De Valck (2016) cita a Bourdieu para explicar ambos conceptos, en sus palabras:

El capital cultural determina el estatus social de una persona en la sociedad (clase) y está formado por el conocimiento, las habilidades, la educación, las actitudes y el gusto. El capital simbólico, finalmente, apunta a los recursos disponibles para uno en base al prestigio, el honor y el reconocimiento. Todas las manifestaciones del capital sirven como "la energía de la física social" (p.105).

Es en los festivales "donde los cineastas pueden adquirir un capital simbólico", formando parte de estas "economías" de la industria cinematográfica, como Hollywood, pero "el principal capital en estos nichos es simbólico: prestigio, honor y reconocimiento" (De Valck, 2016, p.105). Del mismo modo, los festivales de cine han desarrollado un poder institucional, similar al de los museos, donde, por un lado, promueven el cine de autor, pero por otro, tratan de demostrar su poder como "aparatos de representación" (Nichols, 2013, p.38). Para una película, haber sido seleccionada para participar en cualquier sección de un festival de cine implicaría un reconocimiento cultural y un prestigio para la película.

\section{Metodología}

El artículo enfrenta desde una perspectiva histórica el desarrollo del FICValdivia como una plataforma fundamental para el cine chileno contemporáneo. Una de las herramientas primarias es un análisis exhaustivo de sus catálogos desde 1994 hasta 2019 , los cuales son la principal fuente bibliográfica del festival. Junto con eso, se realizó una revisión de prensa que da cuenta de la historia y las películas que han sido parte del festival, tales como Cinechile, Festivalesdecine.cl, El Agente Cine y prensa general. Esta revisión histórica va acompañada de la experiencia de la observación participante, realizada durante los FICValdivia de
2017, como invitado de charlas académicas, y 2019 como prensa especializada.

Para este texto se usaron las entrevistas realizadas de manera online al cineasta Enrique Farías en 2018, a la programadora del FICValdivia Isabel Orellana en 2018, y una conversación vía correo electrónico con la fundadora y ex directora de FICValdivia, Lucy Berkhoff, en 2018. Estas tres conversaciones se realizaron para conocer sobre festivales de cine chilenos con el objetivo de obtener información que no estaba escrita sobre el festival, indagar en sus prácticas curatoriales y explorar la experiencia de los realizadores en el festival.

\section{Orígenes de un encuentro con la cinefilia}

El primer antecedente del FICValdivia data de 1963, cuando, durante la primera escuela de verano de la universidad local, se organizó un ciclo de cine que fue el inicio del cineclub universitario (Rivera-Mayorga, 2006). El Cineclub de la Universidad Austral de Chile (UACh) fue un vínculo entre la universidad y la sociedad valdiviana. Según Lucy Berkhoff, directora del cineclub entre 1987 y 2006 y fundadora y directora del Festival Internacional de Cine de Valdivia desde 1994 hasta 2006, la importancia y trascendencia del cineclub radica en que durante muchos años en Valdivia había escasez de oferta televisiva que la gente suplía asistiendo al cine $^{3}$. Los miembros del cineclub eran principalmente personas vinculadas a la universidad, pero también había integrantes externos a la institución. Durante la dirección de Berkhoff, en 1993, el Cineclub UACh organizó una muestra de cine internacional para conmemorar sus treinta años de existencia. Precisamente a raíz de la celebración de este aniversario que surgió la idea de crear un festival de cine en Valdivia:

Siempre tuve la sensación de que una ciudad pequeña y hermosa como Valdivia merecía un festival de cine para justificar tantos años de trabajo ininterrumpido en el cineclub (...). Además, la presencia de la Universidad le daba un estatus especial. Mi idea era que Valdivia tuviera algo como Cannes, reconocido mundialmente, y que la ciudad se de- 
sarrollara para eventos culturales significativos, construyendo cines, hoteles y desarrollando el turismo (Entrevista con L. Berkhoff, 2018).

En octubre de 1994, se celebró por primera vez la Semana Internacional de Cine de Valdivia. El festival contó desde el principio con el apoyo de la Ilustre Municipalidad de Valdivia, la Universidad Austral y el Ministerio de Educación a través del Fondo para el Desarrollo de la Cultura y las Artes (FONDART), junto al sector privado. El festival presentó dos secciones: Por un lado, una muestra internacional de largometrajes, que contó con ocho películas provenientes de Estados Unidos, Francia, Chile, Perú, Dinamarca y Argentina. Por otro lado, la sección competitiva se dividió entre video, cortometrajes y programas de televisión. La competencia tenía un fuerte énfasis en la ecología y el medio ambiente. En la presentación del festival, Berkhoff definió el evento como un lugar para pensar en el daño ambiental y buscar un equilibrio entre la humanidad y la tierra (Valdivia Cine\&Video, 1994, p.3). El Festival eligió dar al premio principal la forma de un pudú 4 , que se entrega a los ganadores de cada competencia hasta el día de hoy. El primer ganador fue el video Wichan (1994) de Magaly Meneses.

Los primeros cambios significativos del festival comenzaron en 1998. El festival es renombrado como el Festival Internacional de Cine y Video de Valdivia, alejándose de su enfoque ecológico inicial, debido a que tales temáticas dificultaban conseguir patrocinios (Entrevista con L. Berkhoff, 2018). Tal dificultad para financiarse puede entenderse ya que el Fondo Audiovisual no se creará hasta el año 2004, por lo que el Festival dependía de las contribuciones públicas a través de FONDART y de las contribuciones privadas de los patrocinadores. El festival creció en prestigio y relevancia, lo que permitió consolidar el patrocinio de empresas locales, como inmobiliarias, hoteles e industrias madereras.

Al observar algunas de las razones que tienen las ciudades para organizar un festival de cine, el crítico de cine argentino Eduardo Antín (Quintín, 2009) plantea que no tienen necesariamente que ver con el cine, sino más bien con la idea de reunir en una ciudad en un momento determinado gente glamorosa, películas, audiencias y auspicios y patrocinio para agentes culturales. El festival comenzó a instalarse como una alternativa al Festival Inter- nacional de Cine de Viña del Mar. Según Berkhoff, observaron "todo lo que faltaba en Viña del Mar y, luego, Valdivia lo hizo", por ejemplo, al incluir las Escuelas de Cine dentro del festival (Entrevista con L. Berkhoff, 2018). El festival incluía una sección llamada Primer Festival para Niños y Jóvenes, que se centraba en películas para escuelas y hogares infantiles. La identidad que generó el evento en la ciudad implicó una mayor participación de las autoridades locales. El Gobernador de la Región de Los Lagos, el alcalde de Valdivia y el Rector de la Universidad Austral fueron incluidos en el catálogo oficial con breves textos de presentación como signo de que la relación entre el festival y la ciudad se fue robusteciendo con los años.

Para fortalecer el crecimiento del festival, en 2002 Lucy Berkhoff, junto con CORFO y empresarios locales, crean el Centro de Promoción Cinematográfica de Valdivia (CPCV), una organización sin fines de lucro que tiene como objetivo desarrollar y promover el cine de Valdivia (CPCV, 2018). El CPCV comienza a trabajar junto a la Universidad Austral y el Cine Club UACh en la organización del festival, lo que le permitió internacionalizar el festival (Peirano, 2016). Esta forma de organización a través de instituciones sin fines de lucro es una de las más comunes en los festivales de cine a nivel global. Según Rhyne, en general, los festivales "se administran y financian principalmente a través de asociaciones públicas/privadas", creando organizaciones sin fines de lucro, comúnmente denominadas "Tercer Sector", siendo el Estado el "Primer Sector" y el mercado, el "Segundo" (Rhyne 2009, p.10). Para Rhyne, este tipo de organización permite involucrarse en el campo cultural, participando "en el tipo de prestación de servicios y gestión cultural que históricamente ha sido el dominio exclusivo de los gobiernos" (Rhyne, 2009, p.10). Un año más tarde, en 2003, el festival organizó las primeras actividades de la industria, ampliando sus redes más allá de las audiencias y los cineastas, a toda la industria cinematográfica (Castillo, 2013). De la misma manera, el festival también se consolidó como un espacio de debate sobre el cine, con lanzamientos de libros, charlas y talleres abiertos a todo el público.

En 2004 el festival amplió las actividades de la industria, organizando un Work in Progress, junto con la primera mesa de negocios para cine y TV en Valdivia, patrocinada por Pro-Chile y la Asociación de 
Productores de Cine y TV (APCT) y permitió a los participantes tener acceso a distribuidores de Europa y Estados Unidos (FICValdivia, 2004). La creación de espacios para la industria cinematográfica es clave para la consolidación de los festivales de cine. Desde la década de 1980 los festivales de cine han cambiado su relación con la industria cinematográfica. Los festivales de cine "pasaron de ser plataformas pasivas y facilitadoras de la industria cinematográfica a convertirse en intermediarios y actores cada vez más activos en todos los aspectos de la propia industria cinematográfica" (Rüling en Loist, 2016, p.59). En el caso del Festival de Cine de Valdivia, estas actividades lo posicionaron como un espacio indispensable para la industria cinematográfica en Chile. Las actividades de la industria audiovisual se han convertido en fuentes cruciales de financiación y coproducción para las películas, principalmente a través de talleres para cineastas (Vallejo, 2014b). Sin embargo, según Vallejo, los mercados cinematográficos permiten que los festivales de cine se destaquen a nivel internacional:

Los mercados de cine son fundamentales para el impacto internacional de los festivales por el simple hecho de que son una de las principales razones por las que los profesionales de la industria asisten a los festivales en primer lugar. Estos mercados se construyen en torno a las películas en selección en el festival y otros festivales (p.73).

Aunque para Berkhoff el festival del 2006 fue "uno de los mejor realizados" (2018), una ruptura con las autoridades de la Universidad hizo que el equipo organizador del festival renunciara y declinara integrar una nueva junta directiva. La organización del festival, que solía recaer en el cineclub, pasa al CPCV que designó al académico Guido Mutis como nuevo director. En 2007, se incorporaron personas claves para el festival: Raúl Camargo se incorporó al equipo de programación, mientras que Bruno Bettati se hizo cargo de la producción general. Mutis propuso un festival con más intenciones cinéfilas y actividades para construir audiencias. Uno de los cambios introducidos fue la creación de la sección Nuevos caminos, centrada en el cine experimental y los nuevos lenguajes, junto con la invitación de teóricos y críticos de cine como parte del jurado.

Luego de la muerte de Mutis en 2008, el CPVC nombró en 2010 a Bruno Bettati como nuevo direc- tor quien, al mismo tiempo, conservaría el cargo de productor ejecutivo. El festival inicia una etapa de internacionalización y consolidación de sus actividades de industria. Junto al CPCV crean el eslogan Valdivia, Ciudad Cine para construir la imagen de que el cine chileno se celebra en Valdivia y, en 2011, se crea AUSTRALAB, principal instancia de industria que estará en el festival hasta 2017. Este interés por crear una imagen del festival y de la ciudad puede ser abordado desde diferentes perspectivas. Rhyne sostiene que los festivales de cine, para formar parte de una red mundial de distribución de películas, deben estar vinculados a los espacios geográficos donde se celebran:

\begin{abstract}
El festival de cine también está profundamente ubicado en ciudades, regiones y naciones, marcado a través de sus juntas locales de turismo, promovido a través de sus comisiones culturales nacionales y financiado a través de iniciativas supranacionales y fundaciones privadas, con el objetivo de establecer redes internacionales de distribución y exhibición de películas, cuyos esfuerzos subvencionan una industria comercial multinacional del entretenimiento que a menudo busca la manera de explotar mercados locales sin explotar (Rhyne, 2009, p.13).
\end{abstract}

Siguiendo a Rhyne, Wong argumenta que los festivales no sólo celebran el cine, sino también el lugar donde se celebran. Para ella, "los festivales definen el capital cultural que las ciudades y naciones adoptan como eventos de marca para las ciudades de la clase creativa" (Wong, 2011, p.2). Así, los cambios propuestos tenían como objetivo posicionar al festival y a la ciudad como el lugar de encuentro de la comunidad cinematográfica chilena, pero también de una comunidad internacional interesada en el cine chileno. En 2014, Raúl Camargo fue nombrado director. El FICValdivia apuesta a recuperar parte de su origen, incluyendo secciones centradas en el cine indígena y bajo un nuevo lema: Lugar de encuentro e integración (FICValdivia 2014). Basada en una entrevista con Raúl Camargo, Peirano (2016) explica la línea programática del festival como pequeño, arriesgado y experimental.

FICValdivia es conocido en Chile como un evento cinéfilo en el que es posible ver películas realizadas por directores que están comprometidos con el cine de autor y participan en el circuito mundial de festivales de cine. En los últimos años, el festi- 
val ha creado competencias y secciones centradas en narrativas y géneros no habituales en otros festivales de cine en Chile. Nuevos Caminos se centra en la vanguardia y el cine experimental, Disidencias atrae nuevos puntos de vista sobre la resistencia política y social y Primeras Naciones se centra en películas sobre los pueblos indígenas de América Latina. Según Di Chiara y Re (2011), los festivales de cine pueden presentar nuevos puntos de vista sobre el cine de arte contemporáneo, cambiando la perspectiva tradicional de los historiadores del cine (p.135). Con Raúl Camargo al frente, el FICValdivia se ha preocupado por destacar su papel de descubridor de nuevos cineastas y películas.

A partir de 2016, el festival adopta el lema Clásicos del futuro, que se ha convertido en el leitmotiv del festival, desarrollando su imagen en torno a ese concepto. En su sitio web, el festival destaca que su programación "es una programación vanguardista que se anticipa a su tiempo y ofrece un panorama del cine que vendrá, así como la vinculación del cine con otras experiencias y disciplinas" (FICValdivia, 2019). Además, el festival se sitúa como punto de partida para muchos cineastas chilenos que después comienzan a circular en los festivales internacionales ${ }^{5}$.

\section{FICValdivia y su relación con el cine chileno}

Una vez perfilada la trayectoria histórica de la organización del festival, abordaremos la evolución de la programación del cine chileno en ese contexto. En su primera versión, en 1994, el festival estaba enfocado en la ecología: sus tres competencias (video, cortometrajes, programas de televisión), promovían dicha temática. El primer ganador de la competencia principal fue Wichan (1994), dirigida por Magaly Meneses. La película está principalmente hablada en Mapudungun, la lengua mapuche, retratando un juicio mapuche por robo de animales. Entre los años 1994 y 2000, las competencias siguieron enfocadas en cortometrajes. Sin embargo, entre los años 1997 y 2000, se entregó el premio de Mejor Película Internacional a la más votada por la audiencia. En 2003, en conmemoración de los 10 años del festival, se crea por primera vez la competencia de largometraje chileno, resultando gana- dora Cesante (2003), película animada dirigida por Ricardo Amunátegui. Sin embargo, esta competencia desaparece en los años posteriores. Entre 2006 y 2009, el premio al mejor largometraje chileno se entregaba a la película chilena más votada.

Desde la aparición del novísimo cine chileno ${ }^{6}$, en la versión 2005 del FICValdivia, el festival se consolida como la sede de estreno nacional y mundial del cine chileno. Sin embargo, no es sino hasta su versión 2010 que el festival recupera la competencia del cine chileno, ampliando el número de estrenos, permitiendo que sólo dos películas participen en la competencia internacional. En el año 2006, Raúl Ruíz participó en la competencia internacional con Días de Campo (2005), junto con Fuga (2006), ópera prima de Pablo Larraín. Durante ese año, cinco de las 14 películas en las distintas competencias eran chilenas. Algo similar sucede en 2007 cuando sólo dos de las 11 películas en competencia son chilenas. Ese año, la tercera película de Matías Bize, Lo Bueno de Llorar (2006), y el debut de Sebastián Silva, La Vida me Mata (2007), estuvieron en la selección oficial. Ambas películas obtuvieron la Mención Especial del Jurado, mientras que la película de Matías Bize también ganó el Premio de la Crítica. El festival comenzó gradualmente a atraer más películas chilenas en su programación. Según Peirano, desde la década del 2000 el FICValdivia comenzó a tomar la posición que tenía el Festival de Viña del Mar en los años sesenta, como un lugar de encuentro para la cinefilia y donde es posible ver las películas de un nuevo cine chileno emergente. En sus palabras:

\footnotetext{
El festival reúne a algunas de las figuras más prominentes del mundo del cine chileno, y revela los valores y disposiciones del campo local en cuanto a las narrativas culturales del 'cine de arte y ensayo'. Tiene un impacto en la configuración del campo local de la producción cinematográfica, trabajando como guardián del cine de arte y ensayo en el país (Peirano, 2016, p.120).
}

En 2008, Alicia en el país (2008), el primer largometraje del documentalista Esteban Larraín fue la única película en competencia. Durante 2009, se unificaron las competencias de largometrajes y documentales del FICValdivia. De las doce películas en competencia oficial, sólo dos eran chilenas: Turistas (Scherson, 2009) y Noticias (Perut \& Osnovikoff, 2009). La obra premiada como Mejor Pelí- 
cula Chilena se mostró en el foco del cine chileno fuera de competición y fue otorgado a la segunda película de Sebastián Silva, La Nana (2009), una película sobre una trabajadora de casa particular que ve su trabajo amenazado por la llegada de una nueva trabajadora a la casa. La película fue nominada al Globo de Oro a la Mejor Película en Lengua Extranjera, siendo la primera película chilena nominada a estos premios.

Las películas que compiten y ganan premios en el FICValdivia comienzan a circular a nivel internacional y nacional y hay casos en que, incluso, algunas películas son seleccionadas en festivales acredita$\operatorname{dos}^{7}$ después de ser exhibidas en Valdivia. Según Peirano, el FICValdivia es reconocido por la calidad artística de su programación y por ser un "nodo" dentro de la red mundial de festivales:

[FICValdivia] Se ha ganado el prestigio de la comunidad internacional de críticos de cine y programadores de festivales, y sus organizadores tienen conexiones directas con los festivales internacionales más reconocidos que posiblemente programan las películas de arte "más importantes" del mundo del cine contemporáneo (Peirano, 2015, pp. 209-210).

Para muchos cineastas, FICValdivia es una plataforma esencial para la visibilidad e internacionalización de sus películas, siendo una vía de ingreso al circuito internacional de festivales de cine. El cineasta Enrique Farías participó en el FICValdivia en 2014 con su película La madre del cordero (2014). En una conversación con é ${ }^{8}$, relata la importancia del festival en su carrera:

Siempre quise ir al FICValdivia. Al principio, la película iba a ser estrenada mundialmente allí, pero después de ser aceptada en San Sebastián, tuvimos el estreno latinoamericano en Valdivia. Lo importante no fue sólo la exhibición sino también el contacto con otros importantes cineastas, programadores y críticos internacionales, incluso las fiestas. Recibimos muchos comentarios sobre la película por parte de la gente del cine que fue a la proyección, más allá del mero hecho de competir. (Entrevista con Enrique Farías, 2018).

Los festivales de cine pueden entenderse como sitios de paso. De Valck (2007) afirma que son "sitios" porque se realizan en un lugar específico y "de paso" porque tienen una duración temporal (pp.38-
39). Según de Valck, el paso por ciertos festivales les permite a los cineastas "madurar" dentro del circuito internacional. FICValdivia es el sitio de paso principal para una camada de cineastas chilenos desde mediados del 2000 hasta hoy, poniendo énfasis en la búsqueda de nuevos autores, privilegiando los estrenos y las nuevas búsquedas estéticas y narrativas.

La programación de un festival implica el trabajo de un equipo que decide qué tipo de películas se mostrarán en el evento (Frodon, 2010). Raúl Camargo y Guillermo Olivares dirigen el equipo de programación conformado hasta el 2018 por Isabel Orellana (Chile), Gonzalo de Pedro (España) y John Campos Gómez (Perú), encargados de seleccionar las competencias, las galas y algunas de las secciones principales del festival. Isabel Orellana' comentó que la principal dificultad del equipo era la distancia. Ninguno de los programadores tiene el festival como trabajo exclusivo y cada uno vive en ciudades diferentes. Además, comenta que en cada convocatoria abierta reciben diferentes propuestas y que el principal desafío es mantener una identidad curatorial en el festival:

\begin{abstract}
A veces hay películas que son súper valiosas en términos cinematográficos para la industria chilena, pero no necesariamente son películas tan formalmente riesgosas, 0 , no sé, a veces pueden ser un poco más convencionales, y eso también responde a las películas que se están haciendo, así que no puedo inventar las películas chilenas que hay (Entrevista con Isabel Orellana, 2018).
\end{abstract}

Parte de la selección de la competencia del FICValdivia incluye películas que han formado parte del festival en sus actividades de la industria o directores que han participado en el festival con cortometrajes y películas en otras secciones y competencias. Uno de los mejores ejemplos fue la película De Jueves a Domingo (2012), debut de Dominga Sotomayor, que generó expectativas debido al reconocimiento anterior de sus cortometrajes. Su primer cortometraje fue Cessna (2006), que recibió una mención honorífica en el FICValdivia ese mismo año. Su segundo cortometraje, Debajo (2007) ganó la competencia Talento Nacional en SANFIC en 2007, el "Premio Especial del Jurado" en FICValdivia 2007 y recibió premios en otros cinco festivales de Chile, Brasil y España. En 2009, Sotomayor presentó el cortometraje Videojuego (2009) en el FICValdivia ese año, 
que, si bien no ganó ningún premio, fue la primera de sus obras proyectadas en el Festival Internacional de Cine de Rotterdam en 2010.

De Jueves a Domingo se estrenó mundialmente en el IFF Rotterdam en 2012, luego de haber recibido financiamiento del Fondo Audiovisual, el Hubert Bals Fund de escritura y desarrollo de proyecto, y el Hubert Bals Fund de producción. Además de haber sido premiado en AUSTRALAB, Festival de Cine de Biarritz (Francia), Buenos Aires LAB, Ibermedia y haber sido parte de la Cinéfondation Résidence en el Festival de Cannes. La película participó en la competición de los Tiger Awards, ganando el Hivos Tiger Award. En el mismo año, la película obtuvo el Pudú de Oro en la competencia internacional del FICValdivia 2012, la segunda película en ganar esa competencia desde Machuca (Wood, 2004). También, la película recibió un premio especial en la competencia de Cine del Futuro en el BAFICl en 2012. Todos estos festivales también han premiado a la película en su etapa de proyecto. De Valck (2014) sostiene que la participación de una película en estos encuentros de coproducción y mercado da la oportunidad de ser aceptada en el mercado global del cine de arte y ensayo. De Valck también explica cómo los festivales se han transformado en productores de películas: deciden qué película entra o no en el circuito global de festivales de cine. En este caso, la circulación de De Jueves a Domingo permitió asegurar su presencia en los mayores festivales de cine. La película fue premiada en festivales de Chile, Rumania, Rusia, Portugal, Estados Unidos, Bélgica, Canadá, España, Polonia, Países Bajos y Argentina. En Chile, la película participó, además del FICValdivia, en el Festival Internacional de Cine de Iquique (FICIQQ) en 2013, ganando la competencia de cine chileno. Posteriormente, la película se estrenó en las salas comerciales en abril de 2013.

Después del FICValdivia, el futuro de las películas está definido por los contactos y relaciones que los directores y productores establezcan durante el festival. Esto es parte de lo que varios autores han definido como la legitimación cultural de los festivales de cine. Estos certámenes, para presentar nuevos talentos, crean espacios de exhibición que fomentan "la discusión, la reflexión y el compromiso con el contenido y la estética de las películas" (De Valck, 2016, p.106). En el FICValdivia, los competidores de las películas chilenas y algunas de las películas internacionales en competencia están en el festival, presentando cada una de sus películas, asistiendo a las rondas de preguntas después de cada proyección y participando en diversas actividades durante el festival. La forma en que cada uno de los directores interactúe con los demás asistentes al festival dependerá de la red de contactos y amistades tejida en la comunidad cinematográfica chilena.

En 2015, como parte de la competencia internacional, se estrenó mundialmente la película Las Plantas (Doveris, 2015). El largometraje cuenta la historia de Florencia (Violeta Castillo), una adolescente que tiene que cuidar a su hermano que está en estado vegetativo. La película no recibió ningún premio en el FICValdivia. Sin embargo, la visibilidad que tuvo como resultado de competir en el festival la llevó a ser seleccionada en la competencia Generación 14plus en la Berlinale 2016, ganando la Mención Especial del Jurado Juvenil y el Gran Premio del Jurado Internacional Generación 14plus. Así, la película acumuló un prestigio que le permitió formar parte de otros festivales de cine, como el BAFICI (Argentina), Sao Paulo (Brasil) y Jeonju (Corea del Sur).

Otras películas inician un camino hacia su circulación más local. Mala Junta (2016) es el primer largometraje de Claudia Huaquimilla, una directora mapuche que se destacó en los festivales de cine en Chile con su cortometraje San Juan, La Noche más Larga (2012), obteniendo premios en SANFIC, Valdivia y en otros 11 festivales, incluyendo una Mención Especial del Jurado en Clermont-Ferrand en 2013. Mala Junta se estrenó en la competencia nacional del FICValdivia en 2016, ganando el Pudú a la mejor película en la competencia chilena antes de comenzar una amplia circulación en los festivales de cine chilenos. La película recibió premios en casi diez festivales de cine en Chile entre 2017 y 2018. Se estrenó en multicines y salas alternativas el 11 de mayo de 2017, permaneciendo en salas durante 19 semanas, alcanzando 7.321 espectadores (CAEM 2018). Su participación en el FICValdivia permitió que se viera en casi todo el país, siendo elegida por la crítica como una de las mejores películas de 2017. 


\section{Conclusión}

Este artículo analizó el papel que el FICValdivia ha jugado en el desarrollo del cine chileno, enfatizando su evolución histórica y la forma en que exhibe el cine chileno. El FICValdivia se ha posicionado durante más de 25 años como el festival de cine más importante para el cine chileno, permitiendo su internacionalización y creando una imagen del festival que lo inserta como hito fundamental en el circuito mundial de festivales de cine. Todos los años el festival selecciona nuevos autores y nuevas narrativas que surgen en el cine chileno, dando a conocer autores que luego serán promovidos dentro del circuito internacional y dentro del circuito nacional de festivales de cine. Utilizando la terminología de de Valck, el FICValdivia es el principal "sitio de paso" para los cineastas chilenos que buscan internacionalizar sus carreras y películas, insertándose en el circuito global de festivales de cine.

\section{Notas}

1. Todas las traducciones del inglés al español están hechas por el autor.

2. Estas acciones se realizan a través de Laboratorios, Work in Progress, Talent Campus, actividades de Industria, entre otros.

3. Conversación personal con el autor vía correo electrónico (Edimburgo, Reino Unido y Viña del Mar, Chile; entre el 18/06/2018 al 06/08/2018).

4. El pudú (Pudu Puda) es un tipo de ciervo, el más pequeño, autóctono de la zona sur de Chile y Argentina. Su especie está considerada como vulnerable (reducción).

5. Para más información sobre la internacionalización del cine chileno reciente, revisar Peirano, M. (2018). Festivales de cine y procesos de internacionalización del cine chileno reciente. Cuadernos.Info, (43), 57-69. https://doi.org/10.7764/cdi.43.1485.
Además, el festival utiliza la ciudad como lugar de identificación, gracias a sus lemas "Valdivia, Ciudad de Cine" y "Clásicos del Futuro", el festival y la ciudad se posicionan como un punto fundamental para el circuito chileno y latinoamericano, concentrando además la mayor atención de medios de prensa de cualquier festival de cine en Chile. El festival se ha consolidado como el lugar privilegiado para ver cine chileno, las películas participantes se transforman en protagonistas de los circuitos chileno y latinoamericano de festivales de cine, ganando presencia masiva en competencias de festivales menores. Así, muchos de sus autores serán considerados parte de la historia del cine chileno, sus películas serán exhibidas en cineclubes y estudiadas por la crítica y la academia.

6. El término fue acuñado por Gonzalo Maza y Ascanio Cavallo en su libro "El Novísimo Cine Chileno", selección de 21 ensayos sobre 23 cineastas chilenos que compartían ciertos elementos generales en sus películas, y que representan un diferente enfoque al cine chileno desarrollado durante la década de 1990.

7. Festivales acreditados son festivales acreditados por la Federación Internacional de Asociaciones de Productores Cinematográficos (FIAPF), común y erróneamente conocidos como 'festivales clase A".

8. Conversación personal con el autor vía Skype (Edimburgo, Reino Unido y Santiago, Chile; 13/08/2018).

9. Conversación personal con el autor vía Skype (Edimburgo, Reino Unido y Santiago, Chile; 10/09/2018).

10. Se sugiere revisar los informes del festival y el resumen del año del sitio El Agente Cine (www.elagentecine.cl) 


\section{References}

Bourdieu, P. (1993). The Field of Cultural Production. Cambridge: Polity.

CAEM. (2018). El cine en Chile en el 2015. Santiago de Chile: CAEM.

Castillo, P. (2013). Bruno Bettati: "Este año queremos volcar el Festival de Cine a los valdivianos". El Navegable. https://cutt.ly/6yTu1r8

CPCV (Centro Cultural de Promoción Cinematográfica de Valdivia). (2018). CPVC. https://www. cpcr.cll

De Valck, M. (2007). Film Festivals: From European Geopolitics to Global Cinephilia. Amsterdam: Amsterdam University Press.

De Valck, M. (2014). Film Festivals, Bourdieu and the Economization of Culture. Canadian Journal of Film Studies, 23(1), 74-89. https://www.jstor.org/stable/24411693

De Valck, M. (2016). "Fostering arts, adding value, cultivating taste. Film Festivals as site of cultural legitimization". En M. de Valck, B. Kredell \& S. Loist. (Eds.), Film festivals. History, Theory, Method, Practice (pp. 100-116). Londres / Nueva York: Routledge.

Di Chiara, F. \& Re, V. (2011). Film Festival/Film History: The Impact of Film Festivals on Cinema Historiography. Il cinema ritrovato and beyond. Cinémas: Journal Of Film Studies, 21(2-3), 131-151. https://doi.org/10.7202/1005587ar

Doveris, R. (Director) (2015). Las Plantas. [Película]. Chile.

English, J. (2008). Economy of Prestige: Prizes, Awards and the Circulation of Cultural Value. Cambridge, MA: Harvard University Press.

Elsaesser, T. (2005). European Cinema. Face to Face with Hollywood. Amsterdam: Amsterdam University Press.

FICValdivia. (2004). Catálogo Oficial $11^{\circ}$ FICValdivia. Valdivia: publicación propia.

FICValdivia. (2014). Catálogo Oficial $21^{\circ}$ FICValdivia. Valdivia: publicación propia.

FICValdivia. (2019). Catálogo Oficial $26^{\circ}$ FICValdivia. Valdivia: publicación propia.

Koehler, R. (2017). A New Latin Primer. Sight \& Sound, 27 (8), 16-17.

Loist, S. (2016). "The film festival circuit: Networks, hierarchies, and circulation". En: M. de Valck, B. Kredell, y S. Loist (Eds.), Film festivals. History, Theory, Method, Practice (págs. 49-64). Londres y Nueva York: Routledge.

Nichols, B. (2013[1994]). "Global Image Consumption in the Age of Late Capitalism". En: D. Iordanova (Ed.), The Film Festival Reader. St Andrews: St Andrews Film Studies.

Peirano, M.P. (2015). Contemporary Chilean Cinema: Film Practices and Narratives of National Cinema within the Chilean 'Film Community' [tesis de doctorado no publicada]. University of Kent.

Peirano, M. P. (2016). Pursuing, resembling, and contesting the global: the emergence of Chilean film festivals. New Review of Film and Television Studies, 14(1), 112-131. https:// doi.org/10.1080/17400309.2015.1109345 
Peirano, M. \& González Itier, S. (2018). Los Festivales de Cine en Chile (1963/1967-2018). Informe Proyecto Festivales de Cine en Chile: ventanas de exhibición y difusión del cine chileno. Fondo Audiovisual 410942.

Perut, I. \& Osnovikoff, B. (Directores) (2009). Noticias. [Película]. Chile.

Quintín, E. (2009). "The Festival Galaxy". En R. Porton (Ed.), Dekalog 3: On Film Festivals (pp. 38-52). London: Wallflower Press.

Rhyne, R. (2009). "Film Festival Circuits and Stakeholders". En: D. Iordanova y R. Rhyne (Eds.), Film Festival Yearbook 1: The Festival Circuit (págs. 9-22). St Andrews: St Andrews Film Studies.

Rivera-Mayorga, N. (2006). Centro de Desarrollo Audiovisual del Sur [tesis de fin de grado]. Universidad Austral de Chile.

Scherson, A. (Directora) (2009). Turistas. [Película]. Chile.

Valdivia Cine\&Video (1994). Catálogo Oficial. Valdivia: publicación propia.

Valdivia Cine\&Video (1998). Catálogo Oficial. Valdivia: publicación propia.

Vallejo, A. (2014a). Festivales Cinematográficos. En el punto de mira de la historiografía fílmica. Secuencias. Revista de Historia del Cine, (39), 13-42. https://revistas.uam.es/ secuencias/article/view/5838

Vallejo, A. (2014b). Industry Sections: Documentary Film Festivals between Production and Distribution. Iluminace, 26(1), 65-82.

Wong, C. (2011). Film Festivals: Culture, People, and Power on the Global Screen. New Brunswick, NJ: Rutgers University Press.

Wood, A. (Director) (2004). Machuca. [Película]. Chile.

\section{- Sobre el autor:}

Sebastián González es $\mathrm{PhD}(\mathrm{c})$ en Estudios de Cine de la Universidad de Edimburgo. Crítico de Cine y programador en FICIQQ. Este artículo es parte de su tesis doctoral sobre festivales de cine en Chile, financiada por ANID-Becas Chile Folio 72160046.

\section{- ¿Cómo citar?}

González, S. (2020). FICValdivia y su posición en el cine chileno contemporáneo. Comunicación y Medios, (42), 96-107. https://doi.org/10.5354/0719-1529.2020.57288 